\title{
INTEGRATED OPTIMIZATION OF FLOATING WIND TURBINE SYSTEMS
}

\author{
Frank Sandner, David Schlipf, Denis Matha and Po Wen Cheng \\ Stuttgart Wind Energy (SWE) \\ University of Stuttgart \\ 70569 Stuttgart \\ Germany \\ Email: sandner@ifb.uni-stuttgart.de
}

\begin{abstract}
The purpose of this paper is to show an exemplary methodology for the integrated conceptioning of a floating wind turbine system with focus on the spar-type hull and the wind turbine blade-pitch-to-feather controller. It is a special interest to use a standard controller, which is easily implementable, even at early design stages. The optimization of the system is done with adapted static and dynamic models through a stepwise narrowing of the design space according to the requirements of floating wind turbines. After selecting three spar-type hull geometries with variable draft a simplified nonlinear simulation model with four degrees of freedom is set up and then linearized including the aerodynamics with the blade pitch controller in the closed-loop. The linear system allows conventional procedures for SISO controller design giving a theoretically suitable range of controller gains. Subsequently, the nonlinear model is used to find the optimal controller gains for each platform. Finally, a nonlinear coupled model with nine degrees of freedom gives the optimal solution under realistic wind and wave loads.
\end{abstract}

Keywords: Floating wind turbine, integrated conceptual design, blade pitch controller, negative damping, spar platform, reduced floating wind turbine model.

\section{INTRODUCTION}

Offshore wind power is more and more considered a favorable source of renewable energy. High and constant wind speeds allow a stable and very efficient power generation. Whereas fixed-bottom solutions have been installed in Europe offshore wind turbines do not yet exist in other regions. The main reason are steep coasts, which do not permit a fixed-bottom foundation. Research on floating foundations for offshore wind turbines has advanced noticeably during the last ten years. Experience and modeling techniques have been widely adopted from oil and gas industry and adjusted to the specific needs of wind power conversion. Various projects are currently running including the construction of scaled models and full-scale prototypes. While the horizontal axis wind turbine is mostly mounted unchanged on the floating foundation research has not yet agreed on a specific platform type. The most obvious requirement is the hydrostatic restoring, which limits the static pitch angle of the platform and therefore the wind misalignment. In order to reduce the wave excitation of the system the waterplane area needs to be minimized which leads to ballast-stabilized systems. They achieve their stability through ballast instead of a buoyant volume around sea level. Spar-type platforms, however, require either a large metacentric height or a high platform mass. Such a voluminous platform should, preferably, be built out of a more economic material than steel in order to reduce costs. See therefore the concrete solution presented by [1]. For an overview on floating wind turbine (FWT) platforms see [2].

Commonly, a FWT platform is designed by the experts of naval architecture due to the specific engineering background and subsequently assembled with an offshore wind turbine, which is designed separately. Eventually, the blade pitch controller needs to be adjusted in order to fulfil its principle task of maintaining a constant power but also stabilizing the platform dynamics - which, on the other hand, is already defined. Besides the hydrodynamic forces a FWT experiences significant forces on the rotor with their own inherent dynamics. This is why the "coupled" modeling is important. Therefore, the design spiral of an offshore platform, as found in [3] needs to be extended with the wind input and structural dynamics of the wind turbine. The components of the system need to be drafted simultaneously and 
not sequentially.

In this work a methodology shall be outlined to do the conceptual design of a FWT in an iterative and integrated way. For design load case simulations of FWTs, models are mostly nonlinear and in time domain. For early conceptual design and an overall system understanding, however, it is useful to apply reduced order models. Such a model is, e.g., the one described in frequency domain by [4] or the time domain models by [5] and [6]. Two models of a varying level of detail were used for FWT optimization in [7]. One of them is the reduced multibody model presented in [8] with extensions in [9], which is further simplified and linearized in this work. For the hydrodynamic model we first stick to the radiation or maneuvering problem, thus neglecting the wave excitation. By doing so, we can take advantage of the fact that the still water hydrodynamics can be included in the system description, together with the aerodynamics, the structural dynamics and the mooring model, which is eventually entirely linearized for controller design.

The aerodynamic forces on the system depend highly on the wind turbine controller. The controller has the objective of optimizing power output below rated conditions and limit it above in order not to exceed the loads on the drive train components. The controller regulates the aerodynamic torque on the rotor in this high wind speed region by adjusting the blade pitch angle. However, this varying torque also influences the aerodynamic thrust force, which, in turn, significantly impacts the system motion. As has been reported in the literature, there appears a negative damping of the system for a narrow range of the controller gains of the proportional-integral controller. The simplest out of a variety of solutions to this problem is the "adaptation of the controller bandwidth" of a standard PI-controller for the specific FWT platform, see [10] and [11]. The use of an additional controller input has been proposed by [12] and [13]. The complete problem and the variety of possible solutions is nicely compiled in [14].

The methodology presented here focuses on a spar-type platform and its hull and PI-controller optimization for the first drafting of the system. First, three platforms with a determined hydrostatic stiffness and variable draft and diameter are designed. A full linearized closed loop system of equations of a 2D multibody model allows a more detailed look at the platform modes and their alteration with the controller gains. Eventually, we apply the nonlinear model to further tune the gains for each platform before these systems are finally compared to each other in nonlinear simulations with a 9 DOF model under wind and wave excitation.

\section{FLOATING PLATFORM DESIGN}

All three types of floating offshore platforms have already been considered for FWT. The tension leg platform shows fairly different dynamics than the slackly moored systems. However, their characteristics are comparable to already established fixed-bottom offshore structures. The barge with a large buoyant volume around sea level has been considered for FWT but showed limited applicability. The spar platform, on the other hand, avoids the volume around sea level and produces a restoring moment through the distance between the center of volume and the center of mass. This type together with the semi-submersible have been repeatedly studied for the application as FWT platforms. The spar-type platform which is the focus of this paper has been repeatedly applied in the oil \& gas field, although its application for FWTs looks quite different. Spar risers, for example, need to be designed to withstand a variety of payloads with a given eccentricity and not exceed a certain pitch angle for extreme environmental conditions, see [15]. For the wind power application it is far more important to design the hull in order to achieve appropriate coupled dynamics together with the wind power converter. As opposed to an oil \& gas platform the heave motion is not as important, as it is roughly perpendicular to the wind speed. What is most influential is the pitching motion, its resonance and damping. The platforms considered in the following sections are based on steel as hull material, like the first grid-connected FWT prototype "Hywind" and its slightly modified definition for the international code-to-code comparison project "OC3", see [16].

The hydrostatic restoring guarantees that the FWT withstands the thrust forces on the rotor under a limited pitch angle. Thus, the metacentric height alone is not a sufficient measure, since the platform mass determines the restoring together with the distance between the center of buoyancy $z_{C B}$ and the center of mass $z_{C M, F W T}$, see also [15, p.440,f]. The resulting static pitch angle of the platform reduces the power by the factor $c_{\text {pitch, loss }}=\cos ^{3}\left(\beta_{\text {rot }}\right)$, which is the incentive for inventions that compensate the thrust force through a shifting of the ballast within the platform. The hydrostatic stiffness $C_{55}$ does not only include the buoyancy force $\left(\rho g V_{\text {subm }}\right)$ applied at the center of buoyancy at $z_{C B}$ from mean sea level (MSL) and the restoring torque from the waterplane area $\left(\rho g I_{w t r p l n}\right)$ but also all stabilizing and destabilizing gravitational forces of each body $i$ with corresponding center of gravity at $z_{i}$ above MSL of the system. It remains for the hydrostatic restoring stiffness

$$
C_{55}=\sum_{i=1}^{n} z_{i} F_{g, i}+\rho g\left(I_{w t r p l n}-V_{s u b m} z_{C B}\right) .
$$

This value is calculated with the MSL as a reference. Thus, it allows calculating the static pitch angle of the wind turbine under a given thrust force with the hub height $h_{H}$ as lever arm. Figure 1 shows how the spar draft $l_{\text {spar }}$ decreases over the radius while the system mass $m_{F W T}$ increases with the radius for a constant 


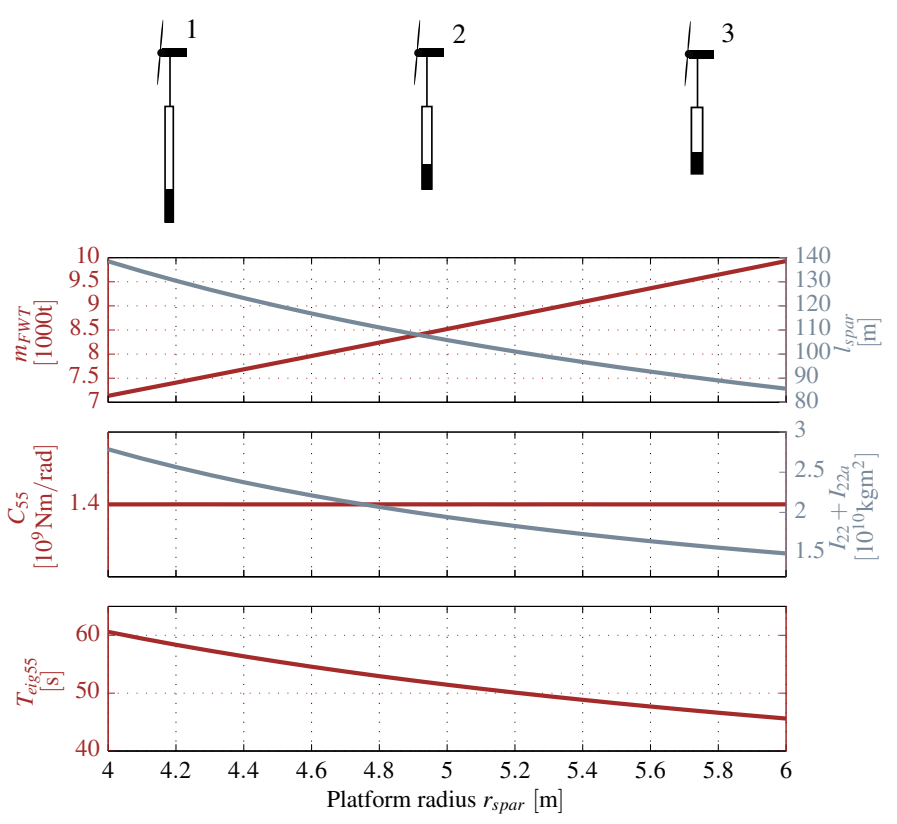

FIGURE 1. Design space of spar platform dimensions with hydrostatic restoring $C_{55}=$ const.

hydrostatic stiffness $C_{55}=1.4 \cdot 10^{9} \mathrm{Nm} / \mathrm{rad}$ for all geometries. The total estimated mass moment of inertia $I_{22}+I_{22 a}$ about $y$ decreases with increasing radii. This estimate includes the additional mass moment of inertia from the added mass with the assumption of an added mass constant of $C_{M}=1$. With this data it is possible to estimate the pitching eigenperiod $T_{\text {eig, } 55}$ of the system about its overall center of mass $z_{C M, F W T}$ with the corresponding stiffness $C_{55, C M}$

$$
T_{e i g, 55} \approx \frac{2 \pi}{\sqrt{\frac{C_{55, C M}}{I_{22}+I_{22 a}}}}
$$

Although the center of the prevailing platform pitching in operating conditions is difficult to determine the overall center of mass is considered a good estimate. The eigenperiod $T_{\text {eig, } 55}$ decreases for increasing radii of the spar. Thus, the platforms with a bigger radius will have a still water eigenperiod that is closer to a common wave spectrum with $T_{p} \approx 10 \ldots 25 \mathrm{~s}$. The sensitivity of the hull to vertical and horizontal Froude-Krylov forces and also the dynamics of the mooring lines are not yet considered here. After this hydrostatic analysis, three representative geometries have been selected out of the design space shown in figure 1, with the characteristics specified in table 1 . These will be used for the following analyses.

\section{CONCEPTUAL DYNAMIC MODELS}

A multibody model of rigid bodies has been set up linked by spring-damper couplings and a simplified representation of the external forces from the mooring lines, the aerodynamic and the hydrodynamic loads. A detailed description of the model principles can be found in [8]. For this work on the coupled floater hull and wind turbine controller design the multibody model has been further simplified. Instead of the number of degrees of freedom $f=9$ we have reduced the number to $f=4$ in order to focus on the most important dynamics and exclude all particular effects that are not of importance at this stage. The fidelity of the multibody model in general has been shown in a comprehensive simulation study with design load case simulations according to the IEC, [17], in [18]. Besides the structure the external force models have also been adapted for this study in a way that the mooring lines are reduced to a single displacement-dependent force in $x$-direction, which exerts on the center of mass of the FWT $z_{C M, F W T}$ in order to exclude the influence of the mooring system as far as possible when comparing the different spar platforms. Consequently, the adjustment of the mooring system will offer the prospect of a further tuning of the system dynamics in subsequent design phases.

We have decided to exclude the wave excitation at this design stage and focus on the radiation or the manoeuvering problem. This is especially advantageous in order to first define the floater hull and the controller since the system including the structure, the simplified mooring lines and the radiation model description can be included in the system equations of motion whereas the aerodynamic forces and the wave excitation cannot be included in this system description. These are terms of control theory and, considering the complexity of the hydrodynamic and wave excitation forces, not an obvious separation. However, in marine craft design it is very common to separate the manoeuvring and the seakeeping problem when analyzing or optimizing a ship layout, see [19]. The therein developed principles and models are very useful for the integrated design and modeling of a FWT.

The radiation problem which assumes still water can be characterized by a frequency-dependent added mass $\mathbf{A}_{\text {add }}(f) \in$ $\mathbb{R}^{6 \times 6}$ and the radiation damping $\mathbf{B}_{\text {rad }}(f) \in \mathbb{R}^{6 \times 6}$, see also [19]. These frequency-dependent quantities are commonly calculated by hydrodynamic panel codes, which are based on potential flow theory. Since this requires rather long calculations with complex software we assume the added mass coefficient to be constant over frequency and equal to the displaced water. This means that the added mass coefficient is, again, $C_{M}=1$, which is a reasonable estimate, see [20]. The radiation damping is modeled as in the initial setup, [8], through the velocity-dependent term of Morison Equation with the translational part $F_{M o r}{ }^{*}, 11$ and the ro- 
tational part $F_{M o r^{*}, 55}$ about the platform center of mass at $z_{\mathrm{CM}, \mathrm{ptfm}}$

$$
\begin{aligned}
& F_{M o r^{*}, 11}=f\left(\dot{x}_{p}, l_{\text {spar }}\right) \\
& F_{M o r^{*}, 55}=f\left(\dot{x}_{p}, \dot{\beta}_{p}, l_{\text {spar }}, z_{\mathrm{CM}, \mathrm{ptfm}}\right) .
\end{aligned}
$$

This is valid for slender cylinders as the ones that are studied here with a predominant influence of flow separation damping. Note that the acceleration-dependent term is already included in the structural equations of motion in order to avoid numeric problems due to the fact that the generalized acceleration is not available as a state. The description of equation (3) depends only on the platform velocity $\dot{x}_{p}$ in translational and $\dot{\beta}_{p}$ in rotational direction. With zero wave kinematics it is possible to solve equation (3) without a numerical integration, which also holds for the mooring line model. This is very useful for obtaining a compact symbolic system description as shown in the section on linearization.

The aerodynamic model that is based on the relative rotoreffective wind speed $v_{r e l}$ and the thrust and power coefficients $c_{P}$ and $c_{T}$ is not explained in detail here but has been described in [8]. The linearization of the aerodynamics in specific will be derived in the section on the linearization of the equations of motion, which follows the next section on the derivation of the nonlinear system equations.

\section{Nonlinear Equations of Motion}

The nonlinear equations of motion of a multibody system of rigid bodies can be set up applying the Newton-Euler equations. Therefore Newton's second law for a translational motion is written for each body as well as Euler's law for rotational motion. The coupled equations of motion result for translational and rotational directions for each body with mass $m_{i}$ and inertia $\mathbf{I}_{i}$ as

$$
\left[\begin{array}{c}
m_{i} \mathbf{E} \cdot \mathbf{J}_{T i} \\
\vdots \\
\mathbf{I}_{i} \cdot \mathbf{J}_{R i} \\
\vdots
\end{array}\right] \ddot{\mathbf{q}}+\left[\begin{array}{c}
m_{i} \mathbf{E} \cdot \overline{\mathbf{a}}_{i} \\
\vdots \\
\mathbf{I}_{i} \cdot \bar{\alpha}_{i}+\tilde{\omega}_{i} \mathbf{I}_{i} \omega_{i} \\
\vdots
\end{array}\right]=\left[\begin{array}{c}
\mathbf{f}_{i} \\
\vdots \\
\mathbf{l}_{i} \\
\vdots
\end{array}\right]+\overline{\mathbf{Q}} \cdot \mathbf{g} .
$$

The upper set of equations are the linear momentum equations with the generalized accelerations $\ddot{\mathbf{q}}$ and the local accelerations $\overline{\mathbf{a}}_{i}$. On the right hand side are all applied forces and moments $\mathbf{f}_{i}$ and $\mathbf{l}_{i}$, respectively and the reaction forces $\overline{\mathbf{Q}} \cdot \mathbf{g}$. The rotational part of the second summand of equation (4) are the Coriolis, centrifugal and gyroscopic forces, which are of importance for FWT due to the rotor dynamics. The local accelerations, marked by the overline, result from the time derivative of the jacobians. The term including the skewsymmetric matrix $\tilde{\omega}_{i}$ results from the time derivative of the rotation matrix that transforms the local mass moment of inertia matrices $\mathbf{I}_{i}$ of each body $i$ into global coordinates. Each line in equation (4) represents the spatial Newton-Euler equations for each body $i=1 \ldots n$, where $\mathbf{E}$ is the unity matrix.

The generalized coordinates are for this simplified model the platform surge $x_{p}$ and pitch $\beta_{p}$ motion, the tower top displacement in platform coordinates, $x_{t}$ and the rotor speed $\dot{\varphi}$ comprised in $\mathbf{q}$ as

$$
\mathbf{q}=\left[\begin{array}{c}
x_{p} \\
\beta_{p} \\
x_{t} \\
\dot{\varphi}
\end{array}\right]
$$

The system motion will be two-dimensional and only in $x-z$ direction, where $x$ is pointing in wind direction, see figure 2 . The platform does not move in vertical direction but only in $x$ and rotate about $y$. The reason for this is that the vertical motion of the platform is not of decisive importance to the system since it is perpendicular to the wind direction. This is a major difference to the modeling of manned oil \& gas platforms, where mostly the first dynamic analyses are addressed to the heave resonances. The formalism for multibody system equations as illustrated in [21] uses generalized coordinates with an absolute description of the kinematics. The rotational and translational jacobian matrices $\mathbf{J}_{T i}$ and $\mathbf{J}_{R i}$ link the absolute motion specified by the position vectors $\mathbf{r}_{i}$ with the curvilinear motion specified by $\mathbf{q}$ via

$$
\mathbf{J}_{T i}=\frac{\partial \mathbf{r}_{i}}{\partial \mathbf{q}} \quad \omega_{i}=\mathbf{J}_{R i}(\mathbf{q}) \cdot \dot{\mathbf{q}}
$$

which holds for scleronomic systems.

We have set up the FWT multibody model for the bodies Platform, Tower, Rotor-Nacelle Assembly (RNA), see figure 2. The procedure specific to the FWT system is described in more detail in [8] and its principle derivation is found in [21].The equations of motion are finally transformed applying the Lagrangian principle in order to eliminate the reaction forces $\overline{\mathbf{Q}} \cdot \mathbf{g}$ in the constrained directions. Subsequently, we bring them into state space for the use with convenient ODE solvers. The nonlinear formulation of the FWT equations of motions reads

$$
\dot{\mathbf{x}}=\frac{\partial \mathbf{x}}{\partial t}=\left[\begin{array}{c}
\dot{\mathbf{q}} \\
\ddot{\mathbf{q}}
\end{array}\right]=\left[\begin{array}{c}
\dot{\mathbf{x}} \\
\mathbf{M}^{-1}(\mathbf{p}-\mathbf{k})
\end{array}\right]
$$

The parameters of the model together with the dimensions of the three selected spars from figure 1 are summarized in table 1 . The wind turbine and tower configuration has been taken 


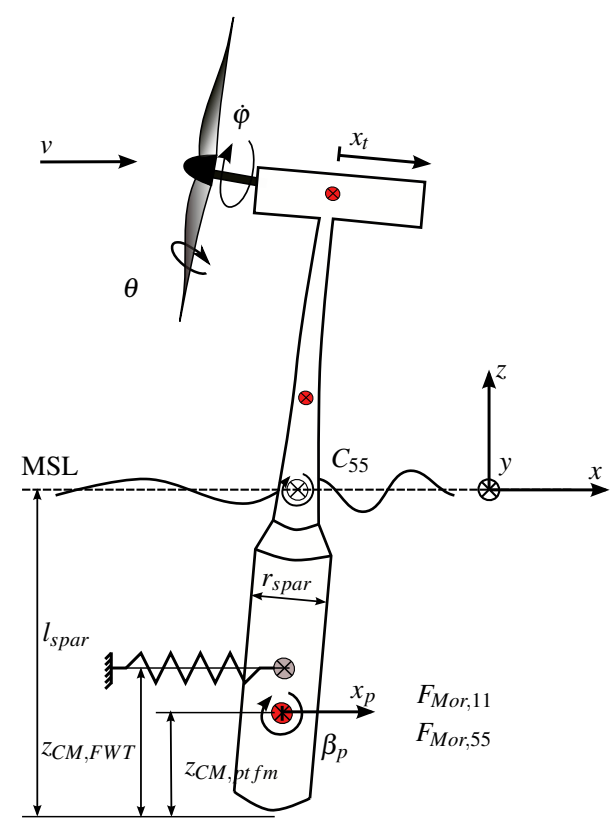

FIGURE 2. Topology of the simplified multibody model.

from the definition of the Offshore Code Comparison Collaboration project (OC3), see [16]. Tower stiffness and structural damping for the equivalent first-order coupling have been identified through free-decay simulations. Also the designed spar platforms are dimensioned with wall thicknesses and ballast densities such that the equivalent diameter matches roughly the one defined by OC3. For simplicity, the reduction of the diameter around sea level has been omitted so that the spar consists of a straight cylinder that pierces the water surface and connects to the wind turbine at $h_{T w r B s}=10 \mathrm{~m}$.

With the nonlinear model described so far and the aerodynamic kinetics described in [8] it is possible to run time-marching simulations with turbulent hub-height wind fields for still water. This model will be used for the controller verification under turbulent winds and extreme operating gusts (EOG). For conventional SISO controller design methods, however, it is necessary to linearize the equations of motion, which is done in the following.

\section{Linearized Equations of Motion}

The nonlinear equations of motion are now linearized around the set point of the states $\mathbf{x}_{0}$

$$
\mathbf{x}=\mathbf{x}_{0}+\Delta \mathbf{x}
$$

TABLE 1. Parameters of the simplified FWT model.

\begin{tabular}{|c|c|c|c|}
\hline Spar radius & $R_{\text {spar }}=$ & {$[4,5,6]$} & $\mathrm{m}$ \\
\hline Spar draft & $l_{\text {spar }}=$ & {$[138.5,105.8,85.7]$} & $\mathrm{m}$ \\
\hline FWT mass & $m_{F W T}=$ & $10^{6}[7.1,8.5,9.9]$ & $\mathrm{kg}$ \\
\hline $\begin{array}{l}\text { Mooring line } \\
\text { stiffness }\end{array}$ & $c_{11}=$ & 41180 & $\mathrm{~N} / \mathrm{m}$ \\
\hline \multirow[t]{4}{*}{ Added mass } & $A_{11, \text { inf }}=$ & $7.1 \cdot 10^{6}$ & $\mathrm{~kg}$ \\
\hline & $A_{55, \text { inf }}=$ & $2.1 \cdot 10^{10}$ & $\mathrm{kgm}^{2}$ \\
\hline & $A_{15, \text { inf }}=$ & 0.0 & $\mathrm{kgm}$ \\
\hline & $A_{51, \text { inf }}=$ & 0.0 & kgm \\
\hline $\begin{array}{l}\text { Morison damping } \\
\text { coefficient }\end{array}$ & $C_{D}=$ & 0.6 & [] \\
\hline
\end{tabular}

where $\Delta \mathbf{x}$ is the new vector of differential states. The same holds for the disturbance, which is here for all analyses the wind speed $v$

$$
v=v_{0}+\Delta v
$$

Ensuing the linearization the coupled equations of motion in state space description from equation (7) can be separated for position- and velocity-dependent terms. It remains with the input $(\mathbf{B} \cdot \Delta u)$

$$
\left[\begin{array}{c}
\Delta \dot{\mathbf{x}} \\
\Delta \ddot{\mathbf{x}}
\end{array}\right]=\underbrace{\left[\begin{array}{cc}
\mathbf{0} & \mathbf{E} \\
-\mathbf{M}^{-1} \cdot \mathbf{Q}-\mathbf{M}^{-1} \cdot \mathbf{P}
\end{array}\right]}_{\mathbf{A}}\left[\begin{array}{c}
\Delta \mathbf{x} \\
\Delta \dot{\mathbf{x}}
\end{array}\right]+\mathbf{B} \cdot \Delta u .
$$

One can identify the position-dependent matrix $\mathbf{Q}$ and the velocity-dependent matrix $\mathbf{P}$, which result from the transformation of the vector of Corliolis, centrifugal and gyroscopic forces $\mathbf{k}$ and the applied forces $\mathbf{p}$ from equation (7). The system matrix $\mathbf{A}$ is now set up for the simplified FWT model. Thus, the equations, calculated by a symbolic programming environment, can now be replaced by the corresponding numeric values after the linear representation of the aerodynamic forces has been set up, which is the focus of the next section.

Linearization of Aerodynamic Kinetics Besides the linearization of the multibody equations of motion the linearization of the aerodynamic power and thrust curves, see figure 3 is quite challenging due to the strong nonlinearity. The curves are 

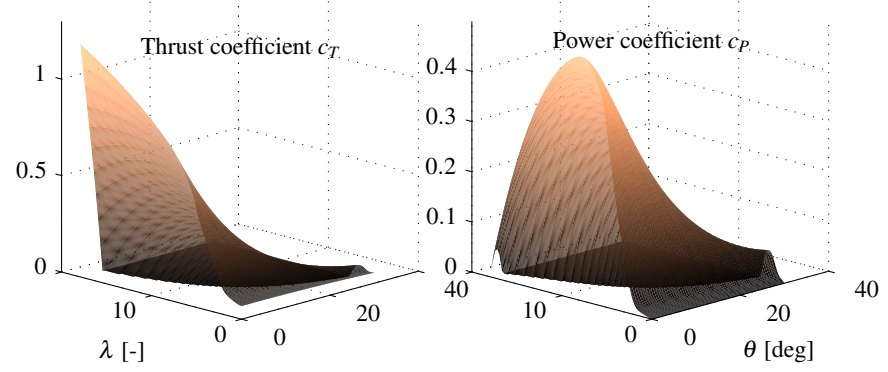

FIGURE 3. Thrust and power curve of the NREL 5MW wind turbine, [22].

extracted from simulations with the NREL 5MW reference turbine, [22], at the steady state with a static wind speed and varying rotor speed $\dot{\varphi}$. The resulting power and thrust coefficient $c_{P}$ and $c_{T}$ is then stored over tip speed ratios $\lambda=\frac{R \dot{\varphi}}{v}$ and blade pitch angles $\theta$. The aerodynamic torque $M_{a}$ acting on the rotor about the shaft axis can be written as a Taylor series up to the second order as

$$
\begin{aligned}
M_{a} & =\underbrace{\frac{\rho \pi R^{2}}{2}}_{k_{\text {aero }}} \frac{v^{3}}{\dot{\varphi}} c_{P}(\lambda, \theta) \\
& \approx k_{\text {aero }}\left[\frac{v_{0}^{3}}{\dot{\varphi}_{0}} c_{P, 0}\right. \\
& +\left(-\frac{v_{0}^{3}}{2 \dot{\varphi}^{2}} c_{P, 0}+\left.\frac{R v_{0}^{2}}{\dot{\varphi}_{0}} \frac{\partial c_{P}}{\partial \lambda}\right|_{0}\right) \Delta \dot{\varphi} \\
& +\left.\frac{v_{0}^{3}}{\dot{\varphi}_{0}} \frac{\partial c_{P}}{\partial \theta}\right|_{0} \Delta \theta \\
& \left.+\left(\frac{3 v_{0}^{2}}{\dot{\varphi}_{0}} c_{P, 0}-\left.\frac{R v_{0}}{2} \frac{\partial c_{p}}{\partial \lambda}\right|_{0}\right) \Delta v\right] .
\end{aligned}
$$

Note that the formulation is specifically written for the power curves as function $c_{P}=c_{P}(\lambda, \theta)$, see figure 3 . The thrust force $F_{a}$ can be written similarly as

$$
\begin{aligned}
F_{a} & =k_{\text {aero }} v^{2} c_{T}(\lambda, \theta) \\
& \approx k_{\text {aero }}\left[v_{0}^{2} c_{T, 0}\right. \\
& +\left.R v_{0} \frac{\partial c_{T}}{\partial \lambda}\right|_{0} \Delta \dot{\varphi} \\
& +\left.v_{0}^{2} \frac{\partial c_{T}}{\partial \theta}\right|_{0} \Delta \theta \\
& \left.+\left(2 c_{T, 0} v_{0}-\left.\frac{R \dot{\varphi}_{0}}{2} \frac{\partial c_{T}}{\partial \lambda}\right|_{0}\right) \Delta v\right]
\end{aligned}
$$

The calculation of the partial derivatives $\frac{\partial c_{x}}{\partial x}$ has been done by applying a central-difference scheme, interpolating linearly between the data points. For a model verification a comparison of the drive train dynamics has been done with a transfer function $G(s)_{v \rightarrow \dot{\varphi}}$ and $G(s)_{v \rightarrow F_{a}}$ calculated by the tool Simulink. It shows that the response depends noticeably on the step size of the central difference method. Eventually, the force $F_{a}$ and the torque $M_{a}$ have been included in the right hand side of equation (4) and were afterwards linearized together with the structural equations of motion to give equation (10).

With the system matrix $\mathbf{A}$ and the corresponding input and output vectors $\mathbf{B}$ and $\mathbf{C}$ the transfer function can be obtained after a Laplace transformation. As system output we have selected the absolute tower top displacement $x_{t, i s y s}$ for an investigation of the impact of the wind input, or the controller on the FWT system. Therefore the output vector takes the form

$$
\mathbf{C}=\left[\begin{array}{llllll}
1 & \left(l_{\text {spar }}-z_{C M, F W T}+h_{h u b}\right) 1 & 0 & 0 & 0 & 0
\end{array}\right]
$$

The transfer functions are calculated for all three selected platforms, defined in table 1 , at a reference wind speed $v_{0}=16 \mathrm{~m} / \mathrm{s}$ with the corresponding steady-state blade pitch angle $\theta_{0}$. The wind speed is chosen for all simulations such that the relative rotor effective wind speed is always in the above-rated wind speed region, also for nonlinear simulations. Figure 4 shows the transfer function from wind speed $v$ to the absolute tower top displacement $x_{t, i s y s}$ for all platforms. The system poles and zeros can be clearly identified: The lowest pole is the eigenfrequency of a predominant platform surge deflection $x_{p}$ at about $\omega_{\text {surge }}=$ $0.045 \mathrm{rad} / \mathrm{s}$. The second resonance is the combined eigenmode with deflections from platform surge $x_{p}$, platform pitch $\beta_{p}$, tower bending $x_{t}$ and rotor speed $\dot{\varphi}$ at about $\omega_{\text {combi }} \approx 0.25 \mathrm{rad} / \mathrm{s}$. The tower eigenmode has an eigenfrequency of $\omega_{\text {tower }} \approx 2.9 \mathrm{rad} / \mathrm{s}$, which is slightly higher than the corresponding eigenfrequency with a fixed tower base $\omega_{\text {tower, onshore }} \approx 2.3 \mathrm{rad} / \mathrm{s}$.

The zero in the right half plane, as also described in figure 6, is the main challenge for the use of the PI-controller for FWT. It results from a negative derivative of the thrust force $F_{a}$ with respect to the wind speed $v$ as $\frac{\partial F_{a}}{\partial v}<0$, see also [14]. It means that there is a frequency at which the platform pitch oscillation is negatively damped due to the characteristics of the thrust curve, see figure 3 .

After calculating the open loop of the system, the feedback control scheme for the blade pitch-to-feather controller will be introduced in order to close the loop.

\section{CLOSED LOOP ANALYSIS OF THE LINEAR SYSTEM}

The controller investigated in this study is the PI-bladepitch-to-feather controller as it is also applied to the OC3 reference spar-type FWT. It maintains a constant generator torque in 


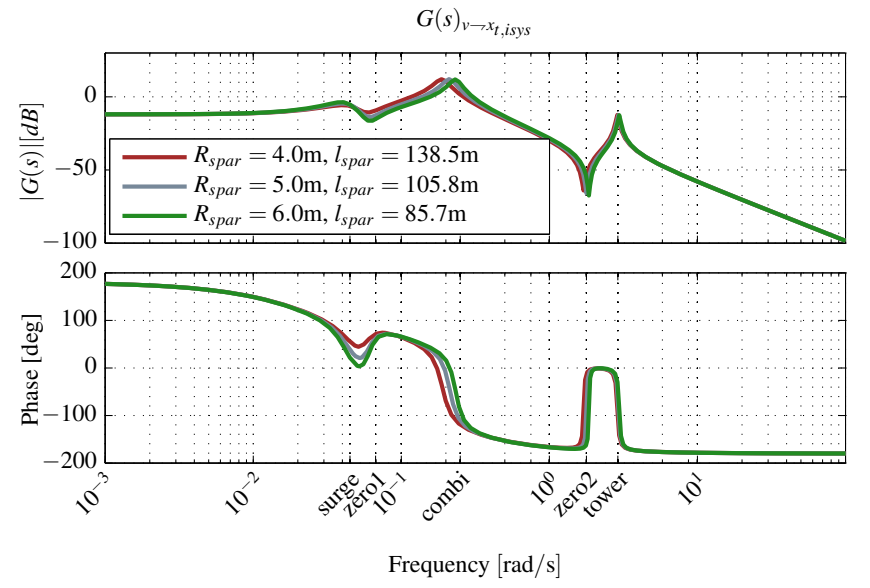

FIGURE 4. Bode plot of the FWT open loop transfer function at $v_{0}=$ $16 \mathrm{~m} / \mathrm{s}$.

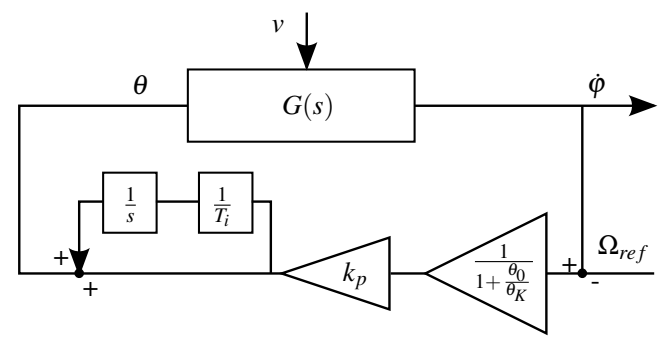

FIGURE 5. PI-controller for above-rated wind speeds.

the above-rated wind speed region. Figure 5 shows the block diagram of the controller. It can be seen that a gain-scheduling block is included in order to adapt the selected gains to the nonlinearity of the power curve, see figure 3 . The function $f_{\text {gain }}=\frac{1}{1+\frac{\theta_{0}}{\theta_{K}}}$ is here adopted unchanged from [16] with a value of the reference blade pitch angle $\theta_{K}=6.3^{\circ}$. The feedback control law can now be transformed into time domain and the blade pitch correction $\left(\theta-\theta_{0}\right)$ written as

$$
\left(\theta-\theta_{0}\right)=\frac{k_{p} f_{\text {gain }}}{i_{\text {gear }}} \Delta \dot{\varphi}+\frac{k_{p} f_{\text {gain }}}{i_{\text {gear }} T_{i}} \Delta \varphi
$$

with the gear ratio $i_{\text {gear }}$.

At this point the complete linear closed-loop system is derived and the eigenvalues of the system or the poles of the transfer function can be analysed. Figure 6 shows the open-loop zeros (o) and the closed loop poles $(\times)$ for the time constant $T_{i}=10 \mathrm{~s}$ and proportional gains $k p=0.004 \ldots 0.05$. It can be seen how mostly the real parts of the eigenvalues vary for different gains $k_{p}$, ex-

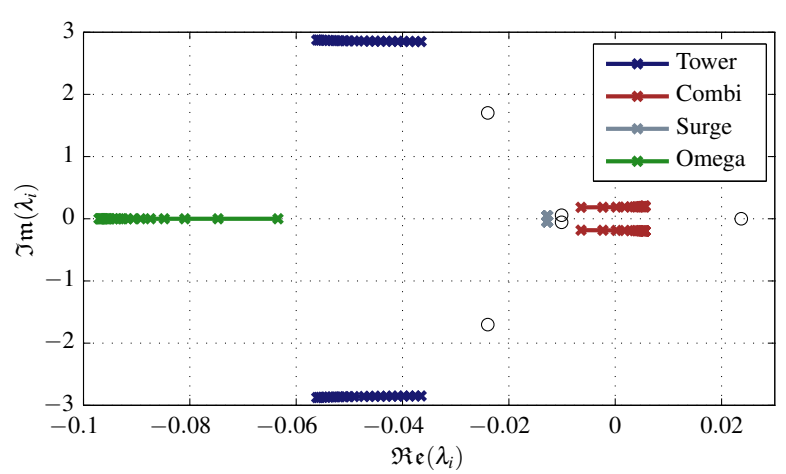

FIGURE 6. Pole-zero map for platform 1 with $k p=0.004 \ldots 0.05$.

cept for the surge mode, which remains fairly constant. It is worth noting that the mode of the azimuth angle $\varphi$ shows increasing real parts for increasing gains, which means that this mode increases its stability margin for increasing $k_{p}$ whereas the tower and combi mode, as mentioned above, show reduced real parts for increasing gains. Consequently, there is a gain at which mainly the coupled combi-mode gets unstable. This crossing into the right half plane should be avoided by all means. The onshore controller achieves its best rotor speed tracking performance for quite high proportional gains and small time constants for which the FWT would already become unstable. Thus, we need to find the best compromise of the rotor speed tracking and the full system stability. A reasonable starting point for this is to select gains at which the coupled platform mode pole is close to the imaginary axis. On such a vertical line in the complex plane the system is evidently stable. Choosing other poles with $\mathfrak{R e}\left(\lambda_{\text {combi }}\right)<0$ further in the left plane would automatically yield a lower real part of the pole related to the rotor and therefore deteriorate rotor speed tracking. It is mentioned that simulations with the nonlinear model have shown that the system performs still fairly well with gains that theoretically yield poles in the right half plane. The fact that a precise determination of the hydrodynamic damping coefficient is in reality not simple will also have to be kept in mind. However, we decided for the further design to take all gains which yield poles on the imaginary axis as possible options.

Figure 7 shows the combined mode poles for different time constants $T_{i}$ and proportional gains $k_{p}$. The imaginary part of this pole increases for increasing $k_{p}$. An increased time constant makes it possible to achieve a stable system with higher proportional gains than with a lower time constant. By applying a root finding algorithm the combinations of $k_{p}$ and $T_{i}$ yielding poles on the imaginary axis are found. It is mentioned that the poles on the imaginary axis of figure 7 will not necessarily have the same damping or the same eigenfrequency. With the design space determined, the nonlinear model will be used in the next section for further optimization. 


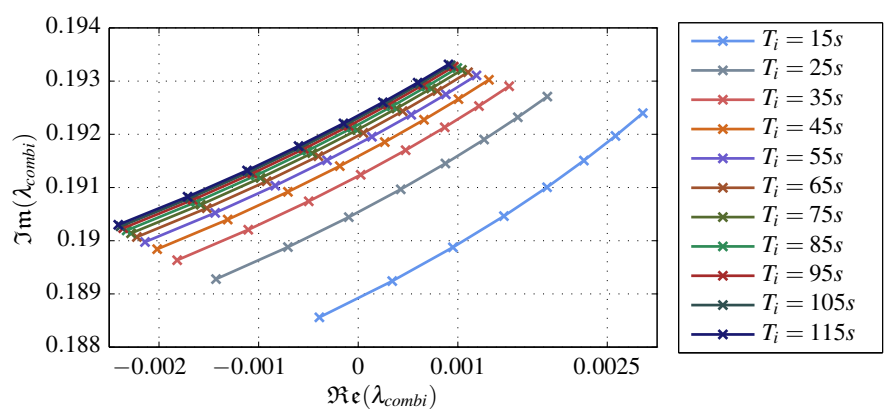

FIGURE 7. Combi mode pole of platform 1 .

\section{OPTIMIZATION WITH NONLINEAR MODEL}

With the design space of the PI-controller determined above the nonlinear model with $f=4$ DOFs will be used with a turbulent wind speed of $v_{0}=16 \mathrm{~m} / \mathrm{s}$ without waves to determine the optimal gains. The selected cost function $J$ includes the two degrees of freedom rotor speed $\dot{\varphi}$ and the tower bending $x_{t}$

$$
J=\sigma^{2}\left(k_{\dot{\varphi}} \dot{\varphi}\right)+\sigma^{2}\left(k_{x_{t}} x_{t}\right)
$$

with the factors $k_{\dot{\varphi}}=10$ and $k_{x_{t}}=1$. With this cost function we are able to assess both, the rotor speed which is the controlled variable and also the platform dynamics. A comparable cost function has already been used in a study that applies the reduced model as internal model for model predictive control, see [23]. Now, not the absolute tower top displacement is used, as before, see figure 4 , but only the degree of freedom of the tower bending $x_{t}$. This is because, generally, the structural loads shall be minimized and not the motion of the system, which is not necessarily coupled. The tower top displacement behaves quite comparably to the tower base moment which is the critical section in terms of stresses. The variance $\sigma^{2}$ is a simple but effective means to boil down the frequency response to a single scalar. For a further comparison of the eventually selected FWT platforms the damage equivalent load will be used.

Figure 8 shows the cost function $J$ for all platforms and the selected range of gains. The optimal gains have been marked with a vertical line. Platform 1 has the highest proportional gain but also the highest time constant. They decrease for the other platforms with a smaller draft and a larger diameter.

The performance in an extreme operating gust of the new developed controllers is shown in figure 9. The solid lines show the results with the adapted controller and the dashed lines show the results for the controller gains defined for the OC3 platform, see [16], which is roughly comparable to Platform 2. The maximum overshoot of the rotor speed is about $15 \%$ of the rated rotor speed $\Omega_{r e f}$. It can be seen that the rotor speed tracking and
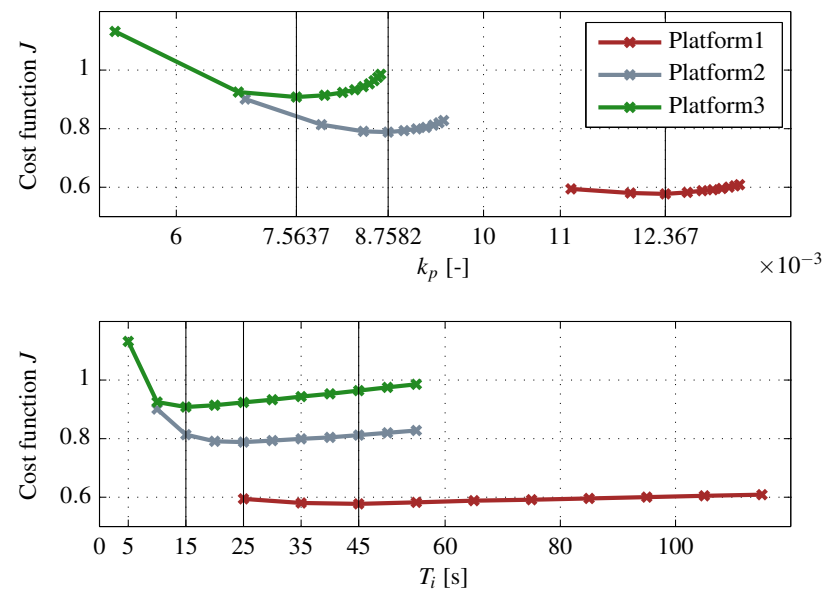

FIGURE 8. Cost function over selected range of proportional gains $k_{p}$ and time constants $T_{i}$.

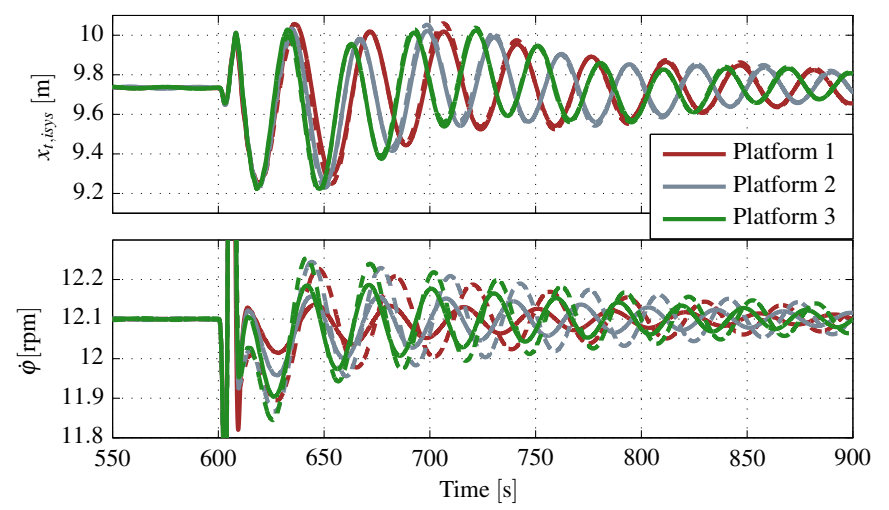

FIGURE 9. Extreme operating gust at $v_{0}=16 \mathrm{~m} / \mathrm{s}$ with OC3 gains (dashed) and optimal gains (solid).

the platform damping is for all platforms better with the adjusted controller gains.

Usually, the controller gains of the PI-controller are chosen by adjusting the bandwidth of the controller. This means that the undamped eigenfrequency of the closed loop of the drive train is compared to the platform eigenfrequency. This method implies a comparison of the closed loop of the uncoupled drive train with the open loop of the rest of the system. However, there is definitely a correlation between the rotor degree of freedom $\varphi$ and the platform and tower degrees of freedom so that a decoupled consideration might be critical. Having this in mind we used our final optimal gains and compared them to the closed loop drivetrain poles. Table 2 shows that for increasing eigenfrequencies 
TABLE 2. Comparison of open loop platform poles with the optimal closed loop controller poles.

\begin{tabular}{cllccc}
\hline Platform & & & 1 & 2 & 3 \\
\hline FWT & $\omega_{\text {combi }}$ & {$[\mathrm{rad} / \mathrm{s}]$} & 0.19 & 0.21 & 0.23 \\
$(\mathrm{OL})$ & $\xi_{\text {combi }}$ & {$[-]$} & 0.108 & 0.094 & 0.087 \\
Drivetrain & $\omega$ & {$[\mathrm{rad} / \mathrm{s}]$} & 0.068 & 0.092 & 0.12 \\
$(\mathrm{CL})$ & $\xi$ & {$[-]$} & 2.55 & 1.9 & 1.47 \\
\hline
\end{tabular}

TABLE 3. Wind wave climate for final selection of FWT.

\begin{tabular}{llccc}
\hline Reference wind speed $v_{0}$ & {$[\mathrm{~m} / \mathrm{s}]$} & 16 & 18 & 20 \\
Significant wave height $H_{s}$ & {$[\mathrm{~m}]$} & 4.3 & 4.7 & 5.1 \\
Peak spectral period $T_{p}$ & {$[\mathrm{~s}]$} & 13.5 & 15.1 & 14.0 \\
\hline
\end{tabular}

of the platform the optimal controller has also increasing eigenfrequencies. The controller damping on the other hand decreases also with the decreasing damping of the platforms. This shows that not only the correlated bandwidth of the controller with that of the platform determines its suitability, but also the correlation of the damping of controller and platform.

So far the spar-type platforms and the corresponding controller gains have been optimized in still water skipping the hull shape optimization in terms of the sensitivity to wave excitation. Nevertheless a final comparison of the three selected platforms will now be performed under turbulent wind and stochastic waves. Therefore turbulent wind fields of IEC Class A with a mean wind speed $v_{0}=16 \ldots 20 \mathrm{~m} / \mathrm{s}$ have been selected in order to make sure that the blade pitch controller acts only in the above-rated regime. Corresponding realistic wave climates were considered from a site of the western North Sea, see table 3. For each wind speed and each FWT model a one-hour simulation has been performed with the full 9 DOF model. This model includes vertical Froude-Krylov forces and the horizontal velocity- and acceleration-dependent terms of Morison Equation. The considered quantities for the evaluation of the FWT performance are on one side, again, the variance of the rotor speed $\sigma^{2}(\dot{\varphi})$ and the damage equivalent load of the tower base as the mean over all single simulations. Figure 10 visualizes the results for all three platforms. The first platform with the largest draft shows the best performance in terms of rotor speed tracking and tower base loads whereas the others perform worse in both. There is a correlation of these results with the open-loop damping of the platform, see table 2, which eventually shows that the hydrodynamic damping of the platform is essential, not only for the tower bending, but also for the rotor speed tracking. On the other hand, the wave excitation loads from the acceleration-dependent term
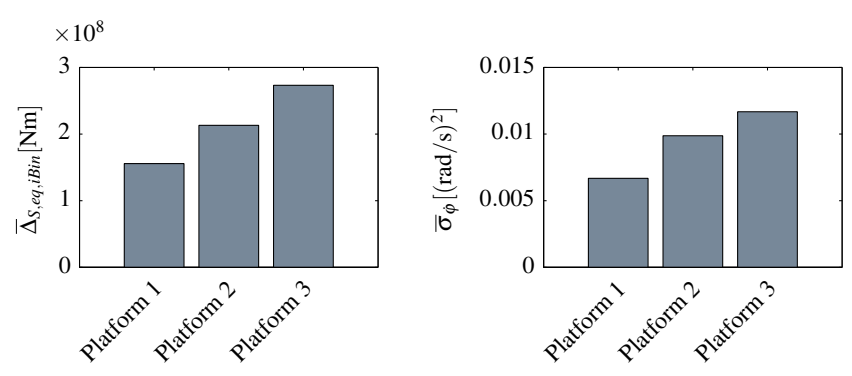

FIGURE 10. Comparison of tower base loads and rotor speed variance for all three platforms.

of Morison Equation and also the vertical Froude-Krylov forces vary to some extent for the spar platforms with their differing draft and radius. Thus, this might also be a reason for the good performance of the deep-drafted spar besides its higher damping. An illustrative overview on the hydrodynamic forces on offshore platforms is presented in [24].

\section{Conclusions}

The study has shown the process of optimizing a floating platform concurrently with the wind turbine controller. The optimal configuration was found through a stepwise narrowing of the design space with three representatively selected spar geometries. Simplified nonlinear and also linearized dynamic models have been developed including a closed loop model with the PI blade-pitch-to-feather controller. The linear models were used to find the stability limit out of the suitable hull shapes for various proportional and integral controller gains. Subsequently, the nonlinear model was used to analyze the frequency response to a turbulent wind excitation and the minimum of a cost function gave the optimal gains for each platform. It could be shown that the controller bandwidth needs to be adjusted for each platform, as stated in previous works, but also the damping has shown to be quite influential and should be taken into account for the controller design. The platform with the largest draft showed the optimal performance under realistic wind and wave loads. Although the maximum depth can be a hard design requirement, this exemplary procedure has proven to be effective and can thus be also applied to other platform types like semi-submersibles. Future work will extent the simplified model with a state-space representation of the radiation problem, see [19], in order to allow for an analysis of non-slender floating platforms.

\section{ACKNOWLEDGMENT}

The work presented in this paper results by one part from the collaborative project "INNWIND.EU" supported by the EU Seventh Framework Program (FP7), grant no. 308974. The second part has been supported by "KIC-InnoEnergy" through the 
project "AFOSP" (Alternative Floating Platform Designs for OffShore Wind Turbines using Low Cost Materials) The financial support is greatly appreciated.

\section{REFERENCES}

[1] Molins, C., Campos, A., Sandner, F., and Matha, D., 2014. "Monolithic Concrete Off-Shore Floating Structure For Wind Turbines". In Proceedings of the EWEA.

[2] de Boom, W., 2011. "Floating Support for Offshore Wind: Small Structures - Big Challenges". In Proceedings of the 30th International Conference on Offshore Mechanics and Arctic Engineering.

[3] Birk, L., Clauss, G. F., and Lee, J. Y., 2004. "Practical Application Of Global Optimization To The Design Of Offshore Structures". In Proceedings of 23rd International Conference on Offshore Mechanics and Arctic Engineering.

[4] Lupton, R., and Langley, R., 2013. "Efficient modelling of floating wind turbines". In Proceedings of 9th PhD Seminar on Wind Energy in Europe.

[5] Betti, G., Farina, M., Marzorati, A., and Scattolini, R., 2012. "Modeling And Control Of A Floating Wind Turbine With Spar Buoy Platform”. In Energy Conference and Exhibition (ENERGYCON), IEEE International, pp. 189194.

[6] Karimirad, M., 2011. "Stochastic Dynamic Response Analysis of Spar-Type Wind Turbines with Catenary or Taut Mooring Systems". PhD thesis, NTNU.

[7] Härer, A., Matha, D., Kucher, D., and Sandner, F., 2013. "Optimization of offshore wind turbine components in multi-body simulations for cost and load reduction". In Proceedings of the EWEA Offshore.

[8] Sandner, F., Schlipf, D., Matha, D., Seifried, R., and Cheng, P. W., 2012. "Reduced Nonlinear Model of a Spar-Mounted Floating Wind Turbine". In Proceedings of the German Wind Energy Conference DEWEK.

[9] Raach, S., Schlipf, D., Sandner, F., Matha, D., and Cheng, P. W., 2014. "Nonlinear Model Predictive Control of Floating Wind Turbines with Individual Pitch Control". In Proceedings of the American Control Conference ACC.

[10] Larsen, T. J., and Hanson, T. D., 2007. "A method to avoid negative damped low frequent tower vibrations for a floating, pitch controlled wind turbine". Journal of Physics: Conference Series, 75.

[11] Jonkman, J., 2008. "Influence of Control on the Pitch Damping of a Floating Wind Turbine". In Proceedings of the ASME Wind Energy Symposium.

[12] Lackner, M. a., and Rotea, M. a., 2011. "Structural control of floating wind turbines". Mechatronics, 21(4), June, pp. 704-719.

[13] Fischer, B., 2012. "Reducing rotor speed variations of float- ing wind turbines by compensation of non-minimum phase zeros". In Proceedings of the EWEA, pp. 144-147.

[14] Veen, G. V. D., Couchman, Y., and Bowyer, R., 2012. "Control of floating wind turbines". In Proceedings of the American Control Conference ACC, pp. 3148-3153.

[15] Chakrabarti, S. Handbook of Offshore Engineering, Volume 1. Elsevier.

[16] Jonkman, J., 2010. Definition of the Floating System for Phase IV of OC3. Tech. rep., NREL.

[17] IEC, 2007. Wind Turbines; Part 3 : Design requirements for offshore wind turbines.

[18] Matha, D., Sandner, F., and Schlipf, D., 2012. "Efficient Critical Design Load Case Identification for Floating Offshore Wind Turbines with a Reduced Nonlinear Model". The Science of Making Torque from Wind.

[19] Fossen, T., 2011. Marine Craft Hydrodynamics and Motion Control, Vol. 1. John Wiley \& Sons, West Sussex.

[20] Sarpkaya, T., and Isaacson, M., 1981. Mechanics of wave forces on offshore structures. Van Nostrand Reinhold Co.

[21] Schiehlen, W., and Eberhard, P., 2004. Technische Dynamik - Modelle für Regelung und Simulation. B.G. Teubner, Stuttgart.

[22] Jonkman, J., Butterfield, S., Musial, W., and Scott, G., 2009. "Definition of a 5-MW Reference Wind Turbine for Offshore System Development Definition".

[23] Schlipf, D., Sandner, F., Raach, S., Matha, D., and Cheng, P. W., 2013. "Nonlinear Model Predictive Control of Floating Wind Turbines". In Proceedings of the Twenty-third International Conference on Offshore and Polar Engineering ISOPE.

[24] Kvittem, M. I., Bachynski, E., and Moan, T., 2012. "Effects of Hydrodynamic Modelling in Fully Coupled Simulations of a Semi-submersible Wind Turbine". Energy Procedia, 24, Jan., pp. 351-362. 